\title{
On the Role of Evaporative Wind in Precataclysmic Binaries (PCB)
}

\section{Pustylnik and V. Pustynski}

\section{Tartu Observatory, Estonia}

Recently we have started a systematic reevaluation of the existing observational methods of analysis hitherto applied to PCB-s. Here we report the first results of our investigations. The improved Napier's algorithm (Napier, 1968) to model the light curves of PCB (with the aid of the set of our computer programs in Turbo Pascal) is used. The source function is taken either from Sobieski (1965) or Strittmatter (1974). The entire luminosity received from the cool companion is calculated by integration of the emerging radiation over its disk; contributions from the illuminated and unilluminated portions of the disk are accounted for. We assume: a) the validity of the LTE in the photosphere of cool component, b) constancy of the monochromatic to mean absorption coefficient ratio within the photosphere, c) the hot star radiates as the absolutely black body, d) there are no other effects influencing the light curve except for the reflection effect. We have modelled the light curves of EC11575-1845 (Chen et al (1995). The analysis of the temperature distributions in the heated photosphere indicates the occurence of the temperature inversion. To make a rough estimate of physical conditions which can induce generation of evaporative wind we have used two criteria: i) the temperature inversion, ii) relation between radiative pressure and the effective gravitational acceleration. We assumed the density varies with the height in the atmosphere of the illuminated star as $\rho \simeq \exp \left(-\Phi / R_{\mathrm{g}} T\right), \Phi$ - being the Roche potential. The integral equation following from the definition of the mean optical depth was solved numerically to establish the relation between the mean optical depth and the distance in the atmosphere. We find the characteristic height scale for X-ray and EUV radiation is $\sim 10^{6}-10^{7} \mathrm{~cm}$ (for concentration of particles $\sim 10^{13} \mathrm{~cm}^{-3}$ ). To check the validity of the ii) criterium we used a simplified model of radially expanding evaporative stellar wind and mass flux $J$ conservation condition along the stream tube of the form $J \sim \rho_{\mathrm{s}} v_{\mathrm{s}} r_{\mathrm{s}}^{2} \exp -\left(\Phi / R_{\mathrm{g}} T\right)$ (Pustylnik (1995)) and found $\dot{M} \sim 10^{-12}-10^{-11} M_{\odot} / y$. Although mass loss of such a rate cannot compete with the effects of the angular momentum loss which is responsible for a secular orbital shrinkage, the evaporative wind should significantly alter the structure of the the cool irradiated components.

\section{REFERENCES}

Chen, A.O.et al, 1995, M.N.R.A.S., 275, No.1, 100.

Napier, W. McD., 1968, Astrophys. Sp. Sci., 2, No1, 61.

Pustylnik,I. 1995, Baltic Astronomy, 4, No.1, 64.

Sobieski, S., 1965, Astrophys. J. Suppl., 12, No.109, 263.

Strittmatter, P. A., 1974, Astronomy \& Astrophys. 32, 7 